\title{
Visual comparison processes: Identity and similarity decisions
}

\author{
JAMES P. CUNNINGHAM \\ Cornell University, Ithaca, New York \\ LYNN A. COOPER \\ University of Pittsburgh, Pittsburgh, Pennsylvania \\ and \\ CELIA C. REAVES \\ Cornell University, Ithaca, New York
}

\begin{abstract}
In the first experiment, in which two successively presented fre-form visual patterns varied in their similarity to each other, subjects had to decide, in one condition, if the patterns were "identical" and in two other conditions if the patterns were "similar." Qualitative individual differences in the effect of similarity on the time required to make a decision were found in the "identity" condition, and these differences interacted with the "similarity" conditions. The individual differences and the experimental effects are interpreted in terms of a two-process model of the visual comparison process-a holistic matching process that is sometimes accompanied by an analytic difference detection process. In the second experiment, the same subjects repeatedly categorized subsets of the free-form visual patterns on the basis of similarity. There appeared to be no individual differences in the subjects' perceptions of similarity, but subjects' perceptions did differ from the assumptions made by the experimenters when they established the response criteria for the first experiment.
\end{abstract}

An important aspect of gaining knowledge about the world is deciding whether two stimuli are the same. The perception of constancies or invariants allows a simplified organization of the world, and thus this visual comparison or perceptual matching process is crucial to our perceptual abilities. In a typical experiment on this process, subjects are repeatedly presented pairs of patterns and asked to decide as quickly as possible if the patterns in each pair are the same or different. A general model of the visual comparison process should be able to explain the relationships between the visual patterns, the decision times, and the error percentages in such an experiment. Even though this process seems relatively simple, a satisfying general model has proved difficult to identify. Nickerson (1972) provides a good review of the models proposed up to that time, and Krueger (1978) provides a more recent model that is impressive in its generality. On the other hand, Cooper $(1976,1982)$ has demonstrated that there are reliable

This work was supported by National Science Foundation Grants BNS-7906253 to the first author and BNS-7622079 to the second author. The results from Experiment 1 were reported at the TwentyFirst Annual Meeting of the Psychonomic Society, St. Louis, November 1980. Kathleen McNellis, Roger N. Shepard, and Stephen E. Palmer provided useful comments on earlier versions of the manuscript. Requests for reprints should be sent to the first author, Department of Psychology, Uris Hall, Cornell University, Ithaca, New York 14853. individual differences in performance in visual comparison tasks and has interpreted these differences in terms of differing processing strategies. The existence of these individual differences suggests that there may not be one generally applicable model of the visual comparison process.

In this paper, we compare the performance of subjects in a task involving the typical same-different or identical-nonidentical decision to their performance in tasks involving a similar-dissimilar decision. We ask whether the individual differences in the way that the identical-nonidentical decision is made are also reflected in the way in which people adapt to the new demands of the similar-dissimilar decision. We attempt to interpret adaptation to this type of decision in terms of a model that can also explain the individual differences between subjects.

\section{Individual Differences in the Comparison Process}

In a series of experiments, Cooper found reliable qualitative individual differences in patterns of data in same-different visual comparison tasks (Cooper, 1976, 1980a, 1980b, 1982; Cooper \& Podgorny, 1976). On a typical trial in one of these experiments, a subject was shown a visual form followed by a second form and was asked to decide if the second form was identical to or different from the initial form. If the forms were different, their similarity in shape could vary from highly similar to highly dissimilar. Cooper 
was able to reliably classify subjects as one of two types, depending upon the pattern of their reaction times in an experiment. For Type I subjects, the speed of the "different" response is not affected by the degree of dissimilarity of a pair of forms, and the "same" response is faster than any "different" response. For Type II subjects, the time required for a "different" response decreases dramatically as the dissimilarity of the pair of forms increases, and the speed of the "same" response is in the middle of the range of the "different" responses. The second type of subject is also slower overall than the first type. While the two types of subjects differ markedly in the pattern of their reaction times, they do not differ in their pattern of errors. The two types of subjects have approximately the same overall percentage of errors, and error percentages decrease with increasing dissimilarity of pairs of forms. These qualitative differences between subjects are reliable in that subjects can readily be classified, and in that a subject remains of a particular type for long periods of time and across several different experiments (Cooper, 1982).

Cooper interprets the difference between these two types of subjects in terms of different processes used to perform the same-different visual comparison. Type I subjects (those whose reaction times are not affected by the degree of dissimilarity of a pair of forms) have been called "holistic processors" and are thought to be comparing a visual memory representation with the second form of the pair in a holistic, perhaps parallel fashion, attempting to find a match between the two representations. If a match is found, they respond "same," and otherwise they respond "different" with no further processing. This predicts the observed insensitivity of "different" reaction times to dissimilarity, since "different" responses are merely made by default. It also accounts for the "same" responses' being the fastest, since "different" is given as a response only after the match process has failed.

Type II subjects (those whose "different" reaction times decrease as the dissimilarity of a pair of forms increases) have been called "analytic processors." They are thought to be using two different processesone an analytic, feature-by-feature comparison process designed to locate the differences between the visual features of two representations, and the other a holistic matching process similar to the one used by the holistic processors. As soon as the analytic comparison process detects a differing feature, a "different" response is given. A "same" response is given if the holistic comparison process finds a match, or if the analytic process does not find a mismatching feature. The holistic process could either occur first, followed by the analytic process only if a match is not found, or the two processes could occur simultaneously. This two-process account is consistent with the pattern of data observed for these subjects. The greater the dissimilarity between two forms, the earlier a differing feature will be found by the analytic process, and thus the faster the "different"' response. The "same" response could be of intermediate speed because some of these responses occur immediately after the holistic match process and some occur after the analytic comparison process. Two-process models of this sort have been proposed by others (e.g., Bamber, 1969) as general models of visual comparison.

Evidence supporting this holistic vs. analytic processing as an explanation of the observed individual differences can be obtained by using experimental tasks that seem naturally to call for the application of one type of comparison process or the other. If a task is most efficiently done by using an analytic comparison process, the subjects who naturally use that process should be able to perform the task easily. However, the subjects who naturally use the holistic process should either have difficulty performing the task or they should switch to the analytic comparison process. Similarly, if a task calls for a holistic comparison process, natural analytic processors should either have difficulty or should switch to a holistic process.

Cooper has obtained evidence of this type from several experiments. For instance, after making a "different" response, most holistic processors are not very accurate in their identification of the location of the difference in shape between the two forms, while analytic processors can accurately locate the difference (Cooper, 1982). This supports the notion that the analytic processors are explicitly detecting differences and thus have access to the location of the differences, while the holistic processors are making holistic matches and thus only have access to matchmismatch information. This is an example of a task that calls for analytic difference detection, and was thus easy for analytic processors but difficult for holistic processors. (See Cooper, 1982, for a discussion of related experiments.)

The studies that will be presented here were designed to test further the holistic vs. analytic processing interpretation of these individual differences in performance in visual comparison tasks. In the first experiment, we asked subjects to expand their "same" response category to include not only pairs of forms that were identical, but also pairs that were very similar. The general idea was to observe the effect on both types of subjects of this change from a decision of identity vs. nonidentity to a decision of similarity vs. nonsimilarity and to see if the effect was interpretable in terms of the holistic vs. analytic processing distinction. In the second experiment, we asked the same subjects to categorize the same forms on the basis of similarity of shape, in order to determine if the two types of subjects differed in their perceptions of similarity and in order to gain informa- 
tion to help in the interpretation of the error rates observed in the first experiment.

\section{EXPERIMENT 1}

In this experiment, we observed holistic and analytic processors in a typical perceptual matching task in which the subjects had to make the distinction between the identity and nonidentity of a pair of forms, and also in a somewhat different task in which the subjects had to make a distinction between similarity and nonsimilarity. One purpose of this experiment was to replicate the performance differences observed in perceptual matching tasks in a new stimulus domain. The other, more important, purpose was to observe precisely how the two types of subjects would respond to the new task demands required by the similarity decision. One possibility was that the holistic processors would find the new task very difficult, because it called for more than just a match-mismatch decision, while the analytic processors would find it easy, because it could be performed by merely altering the criterion number of differing features. If the observed performance effects, whatever their form, can be interpreted in terms of the holistic vs. analytic processing model of individual differences, that will lend support both to our overall account of the perceptual matching process and to our explanation of the observed individual differences.

\section{Method}

Stimuli. The stimuli were the "free form" visual patterns that were originally generated by Shepard and Cermak (1973), a few of which are shown in Figure 1. These forms are generated by a mathematical function defined in polar coordinates. By gradually changing the parameters of the function, the shapes of the forms can be gradually changed. Two parameters of the function were varied, using nine levels of each parameter, thus generating 81 different forms which were identical to the ones used by Shepard and Cermak (1973).

Shepard and Cermak (1973) have demonstrated that as the mathematical parameters of the forms are varied smoothly, the perceived similarity of the forms also varies smoothly. For the shapes in Figure 1, a single parameter is changed step by step to transform the form on the left onto the form on the right. The leftmost form in Figure 1 is one of nine forms designated as "standard," and the other forms can be described by the number of steps in the parameter space separating each from a given standard. The greater the number of steps separating two forms, the greater will be their dissimilarity in shape. The numbers of steps separating two forms is the sum of the number of steps separating the forms on the two dimensions.

The forms were displayed on an oscilloscope screen in a dimly illuminated soundproof booth and appeared as orange patterns on a black background. The forms were approximately $4 \mathrm{~cm}$ in height, and the subject viewed them from approximately $30 \mathrm{~cm}$.

Procedure. The experiment consisted of a sequence of trials, with the subject initiating each trial by pressing either response button. Each trial began with a fixation point displayed on the oscilloscope screen for $1 \mathrm{sec}$; then the point was replaced by one of the nine standard forms, which was displayed for $3 \mathrm{sec}$. After the screen was blank for $1.5 \mathrm{sec}$, another form was displayed. The second form was either identical to the first form or it differed by $1,2,3$, or 4 steps in the parameter space. The subject had to choose the appropriate response as quickly as possible and to indicate that response by pressing one of two buttons. As soon as a response was given, the screen went blank. If the response was incorrect, after a brief pause, the word "error" was displayed for $1 \mathrm{sec}$.

The response that was considered correct depended upon the experimental condition, as depicted in Figure 1. The "identity" (ID) condition was a typical perceptual matching experiment, in which the correct response was "same" if the second form was identical to the first and "different" otherwise. In the "small difference" (SM) condition, the correct response was "similar" if the second form differed from the first by one or fewer steps in the parameter space, with the correct response being "different" otherwise. In the "large difference" (LG) condition, the correct response was "similar" if the second form differed from the first by two or fewer steps and "different" otherwise.

Each subject participated in three sessions on 3 separate days, with one session for each experimental condition. At the beginning of each session, the subject was given instructions and a series
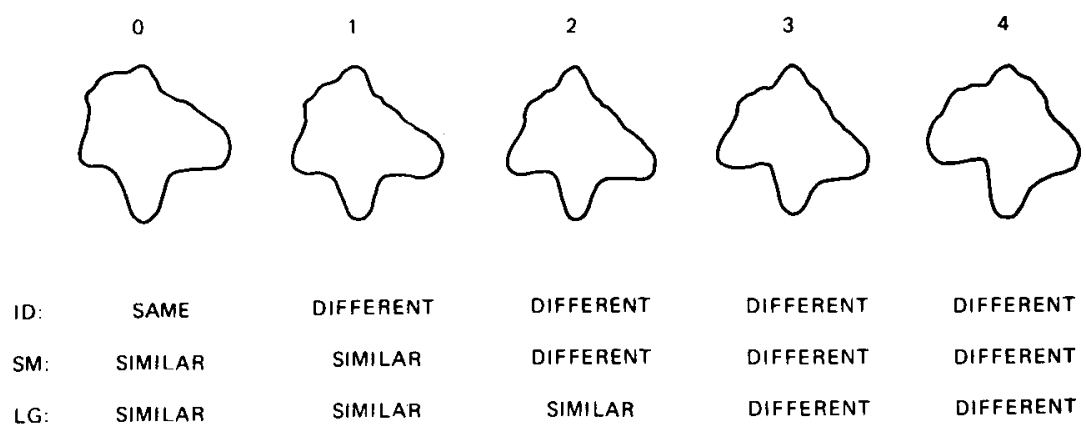
DIFFERENT
SIMILAR
SIMILAR

DIFFERENT

DIFFERENT

DIFFERENT

DIFFERENT

DIFFERENT

DIFFERENT

LG: SIMILAR

SIMILAR

DIFFERENT

DIFFERENT 
of practice trials designed to acquaint him or her with the desired response criterion. The subject then completed 216 test trials from the appropriate experimental condition. On half the trials, the correct response was "different." In each condition, the various numbers of steps of difference for which the correct response was "different" were presented equally often, as were the various numbers of steps of difference corresponding to "same" or "similar."

Subjects. The subjects were students or staff members at Cornell University. Before participating in this experiment, each subject participated in a screening procedure (see Cooper, 1976, for details) in order to determine if the subject was a holistic (Type I) or analytic (Type II) processor. Three subjects of each type participated in this experiment.

\section{Results and Discussion}

The mean reaction time for a correct response is plotted separately for holistic (Type I) and analytic (Type II) processors in Figure 2, as a function of the distance in the parameter space between the first and the second form in a pair. A separate analysis of variance was performed on these reaction times for each type of processor, with the factors being subject, condition (ID, SM, or LG), response ("same/similar" or "different"), and distance $(0,1,2,3$, or 4 steps) nested in the condition $x$ response interaction. The results of these analyses will be reported below as they become relevant.

First consider the results from the ID condition. This condition corresponds to the typical perceptual matching experiment, and we have essentially replicated the results of those experiments. For the analytic (Type II) processors, the time required to respond "different" decreases dramatically with increasing dissimilarity between the two forms, and the "same" response is of intermediate speed. [An analysis of the simple main effect of distance in the ID condition for the analytic processors yields $F(3,18)=12.72$, $\mathrm{p}<.001$.] The holistic (Type I) processors are much faster than the analytic processors, and there is little effect of the dissimilarity between the forms, although there is a slight decreasing trend. [An analysis of the simple main effect of distance in the ID condition for the holistic processors yields $F(3,18)=2.05$, $p>$.10.] This is the expected pattern of individual differences, and demonstrates that the distinction between these two types of subjects is applicable in this stimulus domain.

Now consider the effect on the holistic (Type I) processors of changing the task to one of judging similarity rather than identity. There is no overall effect of condition $[F(2,4)=.44]$ and no interaction of condition $\times$ response $[F(2,4)=1.33]$. However, there does appear to be a slight effect that can be interpreted in the following way. The change in experimental condition can be thought of as requiring a change in the location of the criterion used to distinguish similar stimuli from dissimilar ones. The location of this criterion can be expressed in terms of the number of steps in the parameter space between two forms. For the ID condition this criterion is between 0 and 1 step, while for the SM condition it is between 1 and 2 steps and for the LG condition it is between 2 and 3 steps. If the mean reaction times are replotted as a function of the difference between the criterion and the number of steps separating a pair of

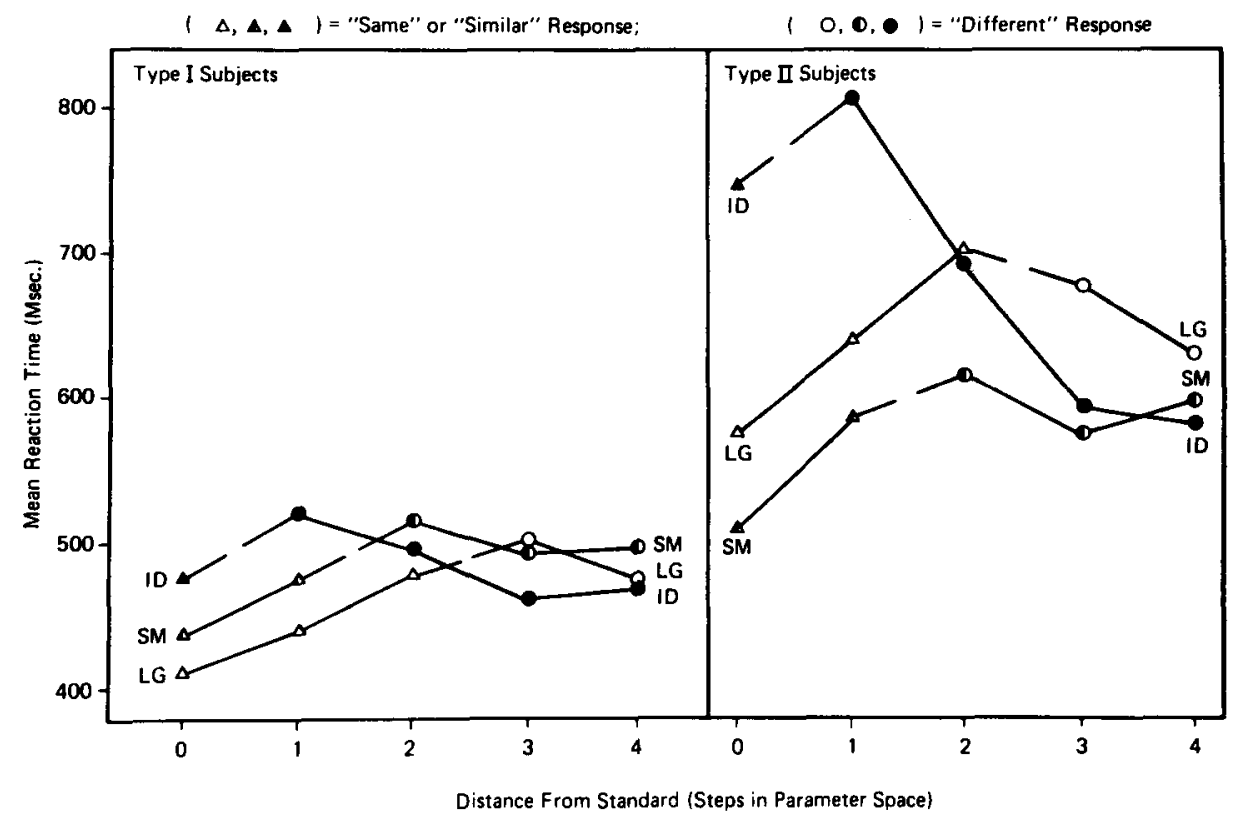

Figure 2. Mean reaction time in Experiment 1 as a function of the dissimilarity between two forms, plotted separately for each condition and each type of subject. The gap in the line for each function represents where the correct response changed from "same" or "similar" to "different." 
forms, Figure 3 results. Note that now all three functions for the holistic (Type I) processors are essentially superimposed. This means that almost all of the variability in the mean reaction times for the holistic processors can be explained in terms of the difference between the dissimilarity between two forms and the criterion dissimilarity for an experimental condition.

These data are consistent with the idea that the holistic processors are using a holistic matching process, but a slight reconceptualization of that process is necessary. It appears that the result of that process is not just a match-mismatch indication, since there is a small overall effect of distance $[F(9,18)=2.50$, $p<.05]$. Rather, the output of the holistic matching process could be an overall measure of the discrepancy between the two forms, analogous to the area of nonoverlap of two templates. That measure must then be compared with some criterion in order to determine the appropriate response. A small amount of time is required to compare the measure with the criterion, and that amount could increase as the measure and the criterion become closer in value. An analogous assumption is often made when considering reaction times in a signal detection task (Pike, 1973). The magnitude of this effect is very small when compared with the effect of distance in the ID condition for the analytic processors, which is consistent with our notion that the two distance effects arise from completely different processes. Thus, our conceptualization is that when a holistic processor is instructed to make a similarity decision rather than an identity decision, he or she changes only the location of the criterion, and the rest of the processing remains unchanged.

Now consider the data of the analytic (Type II) processors (shown on the right in Figures 2 and 3). When these subjects switch from making an identity decision to making a similarity decision, their pattern of data changes dramatically. Overall reaction time is faster for the similarity decision [for condition, $F(2,4)=28.90, p<.01$, and reaction times are much less affected by the dissimilarity between the two forms [for the condition $\times$ response interaction, $F(2,4)=8.26, p<.05$ ]. These data are consistent with our notion that in the ID condition the analytic processors use an analytic difference detection process in conjunction with a holistic matching process. However, when these subjects switch to a similarity decision, they apparently drop the analytic difference detection process, leaving only the holistic matching process. Thus, their performance becomes very similar to that of the holistic processors, who use only the holistic match process in all conditions. A possible reason for the elimination of the analytic difference detection process in the similarity conditions is that the process no longer yields information sufficient for the selection of a correct response, since, in those conditions, even if a difference is located, the correct response could still be "similar." The fact that this switch from a steep reaction time function to a relatively flat reaction time function, along with an overall increase in speed, can be easily interpreted

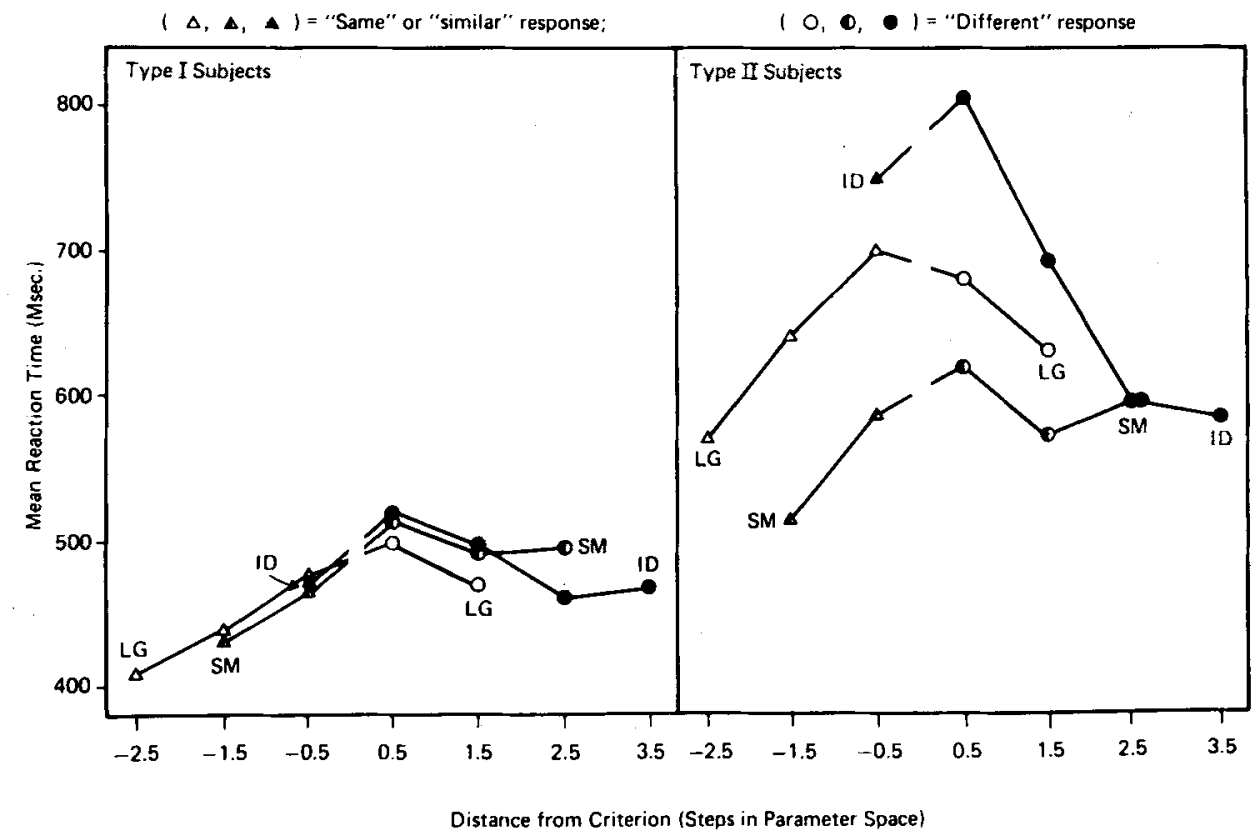

Figure 3. Mean reaction time in Experiment 1 as a function of the difference between the reaponse criterion for each condition and the dissimilarity of the pair of forms, plotied separately for each condition and each type of subject. These are the data from Figure 2 plotted with a different horizontal axib. 
in terms of our two-process model for the analytic processors lends more support to that model.

There are two aspects of the data that pose problems for this interpretation. One is that the analytic processors remain slower than the holistic processors, even in conditions when both types of subjects have flat reaction time functions and are thus thought to be engaging in only holistic processing. The other problematic aspect is that the analytic processors are slower in the LG condition than they are in the SM condition. Given our account of the processing in these conditions, we would expect responses in them to be of approximately equal speed, as in the case of the holistic processors. We have no ready explanation for these two findings.

Now consider the pattern of errors in this experiment. Figure 4 presents the percentage of errors as a function of the dissimilarity between the two forms, plotted separately for the two types of subjects and the three experimental conditions. The pattern of errors is similar for both types of subjects, with the similarity conditions being much more difficult than the ID condition when there are two or more steps separating the forms. Much of this increase in error rate could be caused by subjects having difficulty matching their personal response criteria to the experimenters' imposed criteria, a point that will be addressed by the second experiment. Even though the error rates are very high in some conditions, we have two reasons for thinking that the reaction times for correct responses and the pattern of error rates yield valuable information. One reason is that the pattern of error rates for the ID condition replicates the find- ings of earlier research (e.g., Cooper, 1976) in which reaction times and error rates proved to be very useful. A second reason is that the reaction times for correct responses in conditions which had high error rates are consistent with the reaction times in "neighboring" conditions which had much lower error rates.

In summary, when subjects in a perceptual matching experiment were asked to expand their "same" response category to include not only pairs of forms that were identical, but also pairs that were similar, those subjects responded in a manner that is consistent with our two-process account of the visual comparison process-a holistic matching process that is sometimes accompanied by an analytic difference detection process. If the subject naturally used a holistic comparison process to make the identity decisions, then he or she continued to use that same process in making the similarity decisions, only altering the response criterion. If the subject naturally used a combination of analytic and holistic comparison processes to make the identity decisions, then he or she dropped the analytic process and relied only on the holistic process to make the similarity decisions.

\section{EXPERIMENT 2}

In this experiment, we asked the subjects from Experiment 1 to return and to categorize the same forms on the basis of similarity. One purpose of this experiment was to determine if the subjects that we had classified as holistic or analytic processors also differed in their perceptions of similarity. If they did

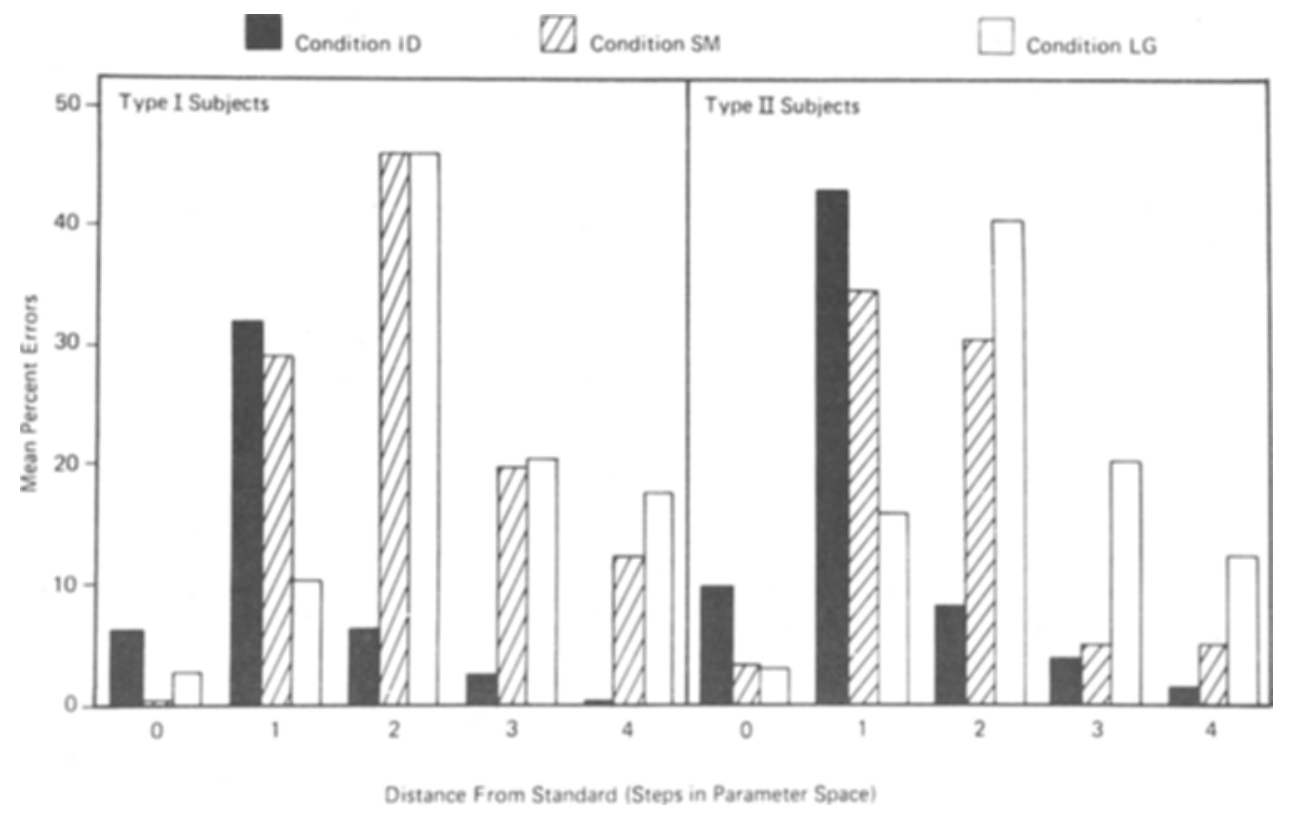

Figure 4. Mean percent errors in Experiment 1 as a function of the dissimilarity between two forms, plotted separately for each condition and each type of subject. 
have differing perceptions, then a one-step parameter difference might not result in equivalent similarity differences for the two types of subjects, and thus their performance differences might be attributed to differing perceptions rather than to the processing differences we have proposed. A second purpose of this experiment was to obtain evidence concerning the relative psychological importance of the two dimensions of the forms' parameter space, and the way in which differences on the dimensions were combined in the perception of similarity. A third purpose was to show that the high error rates in some conditions in Experiment 1 were caused by the difficulty that subjects had in matching their internal response criteria to the experimenters' imposed criteria.

\section{Method}

Stimuli. The 81 forms used in Experiment 1 served as the stimuli for this experiment. The forms varied on two dimensions, each dimension being a parameter of the function used to generate the forms. We will arbitrarily call these dimensions $A$ and $B$, with $A$ being Shepard and Cermak's (1973) dimension $\theta_{2}$ and $B$ being their dimension $\theta_{3}$. Each form was presented as a blue line drawing approximately $6.5 \mathrm{~cm}$ in height centered on a square white card, $13 \mathrm{~cm}$ on a side.

Procedure. Subjects were given nine sets with 41 forms in each set, and instructed to sort the forms in each set into groups of forms that belonged together. They were instructed to make between three and eight groups for each set, but they could use any criteria for deciding what forms belonged in what groups. After sorting each set of 41 forms, the membership of the various groups was recorded. The task took approximately $2 \mathrm{~h}$.

Each of the sets of 41 forms consisted of one of the nine forms designated as a "standard" in Experiment 1, and all of the forms that were no more than four steps in the parameter space away from that standard. The sets were overlapping, with each distinct form appearing in either four or five sets.

Subjects. The subjects were the same six people who had participated in Experiment 1.

\section{Results and Discussion}

Our objective is to examine people's perception of the similarity of pairs of forms used in these experiments. Our derived measure of similarity for a pair will be the proportion of times that the two forms were sorted into the same group, out of all the opportunities that a person had to group those two forms together. The idea behind this measure is that the more similar two forms are perceived to be in shape, the more likely it is that they will be sorted into the same group. Since we are interested in how dimensional differences contribute to the perception of similarity, a pair of forms will be characterized in terms of the number of steps separating the two forms on each of the two dimensions of the parameter space. In this experiment, a pair of forms could differ by from 0 to 4 steps on dimension $A$ or dimension B. Table 1 presents the derived similarities as a function of the separation of a pair of forms on the two dimensions, averaged over all subjects. The proportions in Table 1 decrease monotonically across the rows and down the columns (except for a few
Table 1

Proportion of Times That Two Forms Were Sorted Into the Same Group as a Function of Their Separation on the Two Dimensions

\begin{tabular}{cccccc}
\hline $\begin{array}{c}\text { Steps on } \\
\text { Dimen- } \\
\text { sion A }\end{array}$ & \multicolumn{5}{c}{ Steps on Dimension B } \\
\cline { 2 - 6 } & 0 & 1 & 2 & 3 & 4 \\
\hline 0 & 1.00 & .40 & .11 & .04 & .03 \\
1 & .66 & .36 & .11 & .04 & .03 \\
2 & .44 & .25 & .08 & .05 & .03 \\
3 & .28 & .17 & .06 & .04 & .03 \\
4 & .25 & .13 & .05 & .04 & .05 \\
\hline
\end{tabular}

minor reversals in the very small proportions), which indicates that perceived similarity decreases with an increase in separation on either dimension.

There were three subjects who were natural analytic (Type II) processors and three who were natural holistic (Type I) processors. In order to see if the differences in their patterns of reaction-time data in Experiment 1 could be at least partially attributable to differences in their perception of similarity, the derived similarities for the two types of subjects were analyzed. We performed an analysis of variance on the similarities with the factors of type of subject (holistic or analytic), subject (nested within type), dimension A steps (0 through 4), and dimension B steps (0 through 4$)$. There was not a significant main effect of type of subject $[F(1,4)=1.55]$, but, more importantly, there were no interactions with type of subject [for type $\times$ dimension $A, F(4,16)=.45$; for type $\times$ dimension $B, F(4,16)=2.71$; for type $\times$ dimension $A \times$ dimension $B, F(16,64)=.83 ; p>.05$ for each]. This failure to obtain significance is probably not due to a lack of power in the statistical tests, since the error terms for these tests were also the error terms for tests that reached significance [for dimension $A, F(4,16)=167.21, p<.001$; for dimension $B$, $F(4,16)=266.07, p<.001$; and for the interaction of the two dimensions, $F(16,64)=186.81, p<.001$ ] The fact that there was no interaction between the type of subject and the amount of separation on the two dimensions indicates that the pattern of derived similarities is essentially the same for both types of subjects. Apparently, the reaction-time differences observed in Experiment 1 are not due to differences in perceived similarity, and the group data presented in Table 1 are representative of the perceived similarities of both types of subjects.

Another reason for performing this experiment was to investigate how differences on the dimensions of the parameter space were used in the determination of the similarity between two forms. This involves two related questions: First, what is the mathematical function that combines the differences on the dimensions in order to produce a perception of similarity? Second, what is the relative importance of a 1-step difference on dimension A compared with a 
1-step difference on dimension B? The two questions are related because an answer to the second depends, in part, upon the function used as an answer to the first. Shepard and Cermak (1973) addressed both of these questions, but we address them more formally in an attempt to provide more definitive answers.

Out of the entire range of functions or metrics that could be used to combine dimensional differences, the two that are commonly considered as psychologically plausible are the euclidean and city-block metrics. In order to define these metrics, the importance of a 1-step difference on dimension A will be called $a$, and on dimension $B$ it will be called $b$. The similarity, $s(i, j)$, between two forms that differ by $i$ steps on dimension $A$ and $j$ steps on dimension $B$ will be $s(i, j)=f(a i+b j)$ if the metric is city-block and $s(i, j)=g\left[(a i)^{2}+(b j)^{2}\right]$ if the metric is euclidean, where $f$ and $g$ are unknown monotonically decreasing functions that transform distances into similarities. For seemingly "unanalyzable" stimuli such as the forms used in this experiment, the euclidean metric would be expected to be most appropriate (Attneave, 1962; Garner \& Felfoldy, 1970; Shepard, 1964), but Shepard and Cermak (1973) found some evidence that the psychological metric for these forms was city-block.

If a particular psychological metric is applicable for these forms, then the ordering of the similarities in Table 1 should be consistent with that metric. Only the order information in the similarities is relevant, since the exact forms of the functions $f$ and $g$ are unknown. Before the euclidean and city-block metrics were compared, a few of the smallest similarities were averaged together in order to insure that the similarities decreased monotonically across the rows and down the columns of Table 1 , since both metrics make that prediction. (The method used to choose which similarities should be averaged was multiple monotone regression, as described by Cunningham, in press, or Gebhardt, 1970. This method guarantees that the smallest changes are made in the original data while insuring that the monotonicity condition is satisfied.) Different relative importances of the two dimensions will produce different predicted similarity orderings, but if a particular metric is applicable, there should be a value of the ratio of the dimensional importances, $a / b$, which produces a predicted similarity ordering identical to the observed ordering. In order to evaluate each metric, we examined the constraints on the ratio $a / b$ that are imposed by the observed ordering of the similarities in conjunction with that metric. For the euclidean metric, if $.354 \leqslant a / b \leqslant .378$, then the metric fits the similarities perfectly. For the cityblock metric, there is no value of $a / b$ for which the metric fits perfectly, but the value that comes closest is $\mathrm{a} / \mathrm{b}=.250$, for which there are only two small discrepancies $[s(1,1)=.36$ should be .32 and $s(3,0)=.28$ should be .32]. Thus, we found that the euclidean metric seems to describe best how the differences on the dimensions are combined in order to produce a perception of similarity, and also that a 1-step difference on dimension $B$ is equivalent to a difference of approximately .37 on dimension $\mathrm{A}$.

Both of these findings are at variance with the findings of Shepard and Cermak (1973). They informally estimated that a 1-step difference on dimension B was equivalent to a .5 difference on dimension $A$, and, based on that estimate, it appeared that the city-block metric fit their data better than did the euclidean. We addressed the questions more formally than they and obtained different answers, but our answers are also tentative, since the euclidean metric fit our data only slightly better than the city-block metric. It does, however, seem more plausible that the metric is euclidean, since, if it were city-block, that would imply that the dimensions $A$ and $B$ of the parameter space were the psychologically meaningful dimensions of the set of forms rather than just arbitrary mathematical parameters varied in order to produce the forms. (The reason that this would be true is that a rotation of the dimensions will not change the distances between forms if the metric is euclidean but will change the distances if the metric is cityblock.)

The results of Experiment 2 can also be used to show that the high error rates in some conditions in Experiment 1 were probably caused by the difficulty that subjects had in matching their own personal response criteria to the experimenters' imposed criteria. When we established the criteria for distinguishing similar pairs from different pairs in the SM and LG conditions of Experiment 1, we were implicitly using the city-block metric and equal weighting of the dimensions. We have just seen that, in the subjects' perceptions of similarity, the dimensions were not weighted equally, and a euclidean metric seemed to provide a better description. This mismatch between the way in which the experimental conditions were set up and the subjects' perceptions of similarity could have made it difficult if not impossible for the subjects to establish correct response criteria in the similarity conditions of Experiment 1.

In order to see more precisely if the high error rates could be explained by this mismatch, we examined each subjects' perceived similarities in Experiment 2 and determined for each subject what the similarity criteria were that would produce the fewest errors in the similarity conditions of Experiment 1. Figure 5 presents, for each subject, similarity measures, imposed response criteria, and response criteria that would lead to optimal performance. There is a diamond-shaped set of digits for each subject, with the location of each digit corresponding to the number of steps in each direction along each dimension that separated a form from the "standard" form in its set. The central digit corresponds to the "stan- 

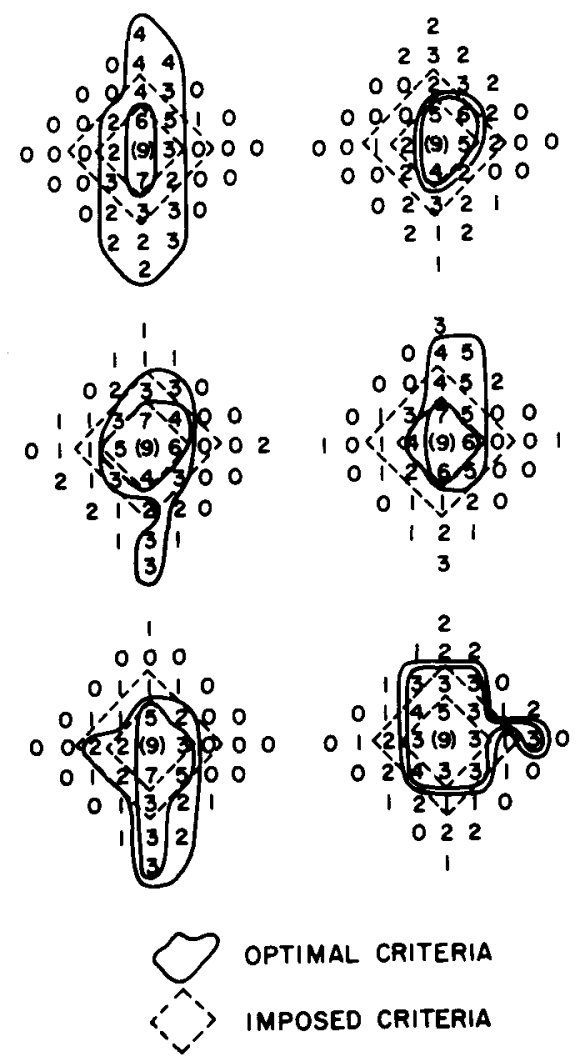

Figure 5. Measures of the similarity of the "standard" form to each of the 41 types of forms in a set as determined in Experiment 2, presented separately for each subject. Superimposed upon this are the response criteria imposed by the experimenters in Experiment 1 and the similarity-based response criteria that would have led to optimal performance in Experiment 1 for each subject. The text contains a more detailed description.

dard" form. Each digit indicates the number of times that a form represented by that location was sorted into the same group as the "standard" form, and can be used as a measure of the similarity between it and the "standard." The central digit is 9 in each case because there were nine sets of forms and the "standard" always had to be sorted with itself. The dashed contours indicate the response criteria imposed by the experimenters in Experiment 1. The inner contour corresponds to the SM condition, and the outer contour corresponds to the LG condition, with the correct response being "similar" for the forms inside a contour and "different" for the forms outside a contour. In order to respond accurately, a subject in Experiment 1 had to adopt these response criteria. However, if a subject based his or her responses only on the perceived similarity of the pair of forms presented on a trial, and if the frequencies presented in Figure 5 are adequate measures of perceived similarity, then completely accurate responding would evidently be impossible. For each subject we established (by exhaustive search) the criteria based on that subject's own similarities that would have led to the lowest error rates in Experiment 1. These optimal criteria are indicated by the solid contours in Figure 5, once again with the inner contour corresponding to the SM condition and the outer contour corresponding to the LG condition. Note that the optimal criteria do not generally coincide with the imposed criteria, with the optimal criteria tending to be elongated along the vertical dimension $B$ and shortened along the horizontal dimension $\mathbf{A}$.

Table 2 reports the observed error percentages and the averaged percentages predicted by the optimal response criteria for the SM and LG conditions. In theory, if the subjects' perceptions of similarity were accurately determined in Experiment 2, then the lowest error percentages that the subjects could have achieved in Experiment 1 would be these optimal percentages. These optimal percentages range up to .48 , indicating that for some separations, error-free performance was virtually impossible. The optimal error percentages show the same general pattern as the observed percentages, with the highest values being for the intermediate-sized separations. Thus, the high error rates observed in Experiment 1 can largely be attributed to a mismatch between the subjects' perceptions of similarity and the assumptions made by the experimenters when response criteria were established. The observed error percentages are, however, higher than the optimal ones in all but one case, indicating that there are also other factors that contribute to the observed error rates.

In summary, Experiment 2 demonstrates that holistic and analytic processors do not differ in their perceptions of similarity for these forms, and therefore the performance differences observed in Experiment 1 cannot simply be attributed to differing perceptions. However, subjects' perceptions of similarity do differ from the assumptions made by the experimenters when they established the response criteria for Experiment 1. Differences on dimension A are weighted much more than are differences on dimension B in subjects' perceptions of similarity,

Table 2

Observed and Optimal Mean Percentage of Errors for Experiment 1 as a Function of the Separation of the Two Forms in the Parameter Space

\begin{tabular}{lccccc}
\hline & \multicolumn{5}{c}{ Steps in Parameter Space } \\
\cline { 2 - 5 } & 0 & 1 & 2 & 3 & 4 \\
\hline \multirow{5}{*}{ Observed } & .02 & .32 & .38 & .12 & .09 \\
Optimal & .00 & .21 & .19 & .06 & .01 \\
& \multicolumn{5}{c}{ Condition LG } \\
Observed & .03 & .13 & .44 & .20 & .15 \\
Optimal & .00 & .04 & .48 & .19 & .09 \\
\hline
\end{tabular}


and this mismatch between the experimenters' assumptions and the subjects' perceptions apparently resulted in high error rates in some conditions of Experiment 1 .

\section{GENERAL DISCUSSION}

The systematic qualitative individual differences that are observed in the pattern of reaction times in typical perceptual matching experiments, and that were observed in Experiment 1, can be attributed to processing differences among the subjects. There appear to be two different kinds of comparison processes available. One is a holistic matching process, in which one visual form is compared with a memory representation of the other form in a rapid, holistic, perhaps parallel fashion. The other is an analytic comparison process, in which a systematic search is made for the location of the differing features of two representations. One type of subject, termed a holistic processor, appears to use only the holistic matching process, whether the required decision is one of identity vs. nonidentity or one of similarity vs. dissimilarity. The other type of subject, termed an analytic processor, appears to use a combination of holistic and analytic processes when making an identity decision, but relies on only the holistic process when a similarity decision is required. The results from the second experiment suggest that the performance differences observed in Experiment 1 should be attributed to processing differences, such as those just outlined, and not to differences in the perception of similarity.
Several other investigators have performed experiments in which subjects made identity decisions in one condition and similarity decisions in another (Bamber, Herder, \& Tidd, 1975; Derks, 1972; Sekular \& Abrams, 1968; Silverman \& Goldberg, 1975; Taylor, 1976). For these investigators, the switch to the similarity decision did not result in an increase in the speed of response and a decrease in the effect of dissimilarity, which is the overall effect that we would have observed in Experiment 1 if we had averaged over both types of subjects. Instead, previous investigators obtained results similar to those displayed in Figure 6, in which the data of Sekular and Abrams (1968) and Taylor (1976) have been replotted in a manner analogous to our Figure 2 . In all of these studies, the similarity decision resulted in a decrease in the speed of response, an increase in the effect of dissimilarity, and a change in direction of the effect of dissimilarity, with higher dissimilarity resulting in longer response times in the similarity conditions. In fact, the perceptual matching model of Krueger (1978) predicts exactly this pattern of data, and that fact is used to support his model.

The reason that we obtained such strikingly different results lies in the differing nature of our stimuli and our task. In all of the other studies, the stimuli consisted of the concatenation of a fixed number of discrete elements-strings of letters or digits, patterns consisting of a small number of line segments, or patterns of black and white squares-and the similarity condition consisted of distinguishing pairs that had no elements in common from pairs that had at least some elements in common. Under those condi-

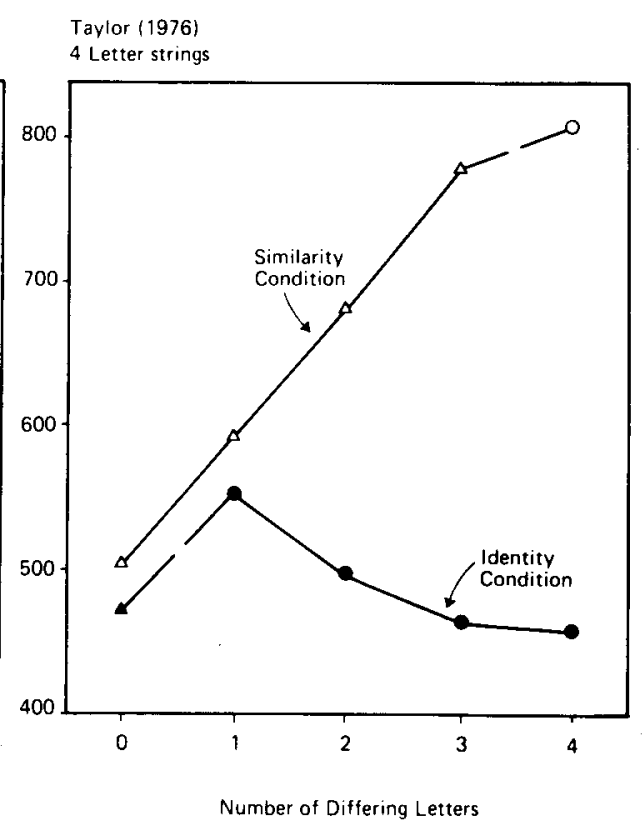

Figure 6. Mean reaction time as a function of the dissimilarity between two stimuli, plotted separately for the similarity and identity conditions of Sekular and Abrams (1968) and Taylor (1976). 
tions, a reasonable strategy would be to scan serially each element of one stimulus, checking to see if that element was a part of the other stimulus, and to respond "different" only if no elements in common were located. That strategy would produce the pattern of data shown in Figure 6. Our study differed in two important respects: the stimuli were relatively unanalyzable blob-like forms that varied continuously in shape, and our similarity conditions required distinguishing similarity from dissimilarity, rather than "some similarity" from "no similarity." With our stimuli and our task, a simple serial scanning strategy would not be effective, so, instead, subjects apparently relied exclusively on a holistic comparison process in the similarity conditions. Krueger (1978) does not explain the results of similarity conditions in terms of a switch to a serial scanning strategy, but instead shows that results such as those in Figure 6 would naturally result from his model. Whether Krueger's model can also naturally account for the results from our similarity conditions remains to be seen.

We have presented a general description of the visual comparison process-a holistic matching process that is sometimes accompanied by an analytic difference detection process. This description can account for the individual differences observed in typical perceptual matching experiments, with some subjects using only the holistic matching process and others using both processes. It can also account for most of the changes in performance that are observed when the matching task is changed to one of judging similarity. Apparently, the observed performance differences are not caused by differing perceptions of similarity, but rather by different processing strategies that are naturally employed by the subjects. This research illustrates a general approach to the study of cognition in which differences between people are used as an aid in the development of theories. This approach enables the researcher to recognize the flexibility of the human mind and still develop general process theories.

\section{REFERENCES}

Attnenve, F. Perception and related areas. In S. Koch (Ed.), Psychology: A study of a science (Vol. 4). New York: McGrawHill, 1962.
BAmber, D. Reaction times and error rates for "same"-."different" judgments of multidimensional stimuli. Perception \& Psychophysics, $1969,6,169-174$.

Bamber, D., Herder, J., \& Tidd, K. Reaction times in a task analogous to "same" ".different" judgment. Perception \& Psychophysics, 1975, 18, 321-327.

COOPER, L. A. Individual differences in visual comparison processes. Perception \& Psychophysics, 1976, 19, 433-444.

Cooper, L. A. Spatial information processing: Strategies for research. In R. Snow, P.-A. Federico, \& W. E. Montague (Eds.), Aptitude, learning, and instruction: Cognitive process analyses. Hillsdale, N.J: Erlbaum, 1980. (a)

Cooper, L. A. Recent themes in visual information processing: A selected overview. In R. E. Nickerson (Ed.), Attention and performance (Vol. 8). Hillsdale, N.J: Erlbaum, 1980. (b)

Cooper, L. A. Strategies for visual comparison and representation: Individual differences. In R. J. Sternberg (Ed.), Advances in the psychology of human intelligence. Hillsdale, N.J: Erlbaum, 1982.

Cooper, L. A., \& Podgorny, P. Mental transformations and visual comparison processes: Effects of complexity and similarity. Journal of Experimental Psychology: Human Perception and Performance, 1976, 2, 503-514.

Cunningham, J. P. Multiple monotone regression. Psychological Bulletin, in press.

DERKs, P. L. Visual recognition of similarity and identity. Journal of Experimental Psychology, 1972, 95, 237-239.

GarNE R, W. R., \& Felfody, G. L. Integrality of stimulus dimensions in various types of information processing. Cognitive Psychology, 1970, 1, 225-241.

GEBHARD', F. An algorithm for monotone regression with one or more independent variables. Biometrika, 1970, 57, 263.271.

Knueger, L. E. A theory of perceptual matching. Psychological Review, 1978, 85, 278-304.

Nickerson, R. S. Binary-classification reaction time: A review of some studies of human information-processing capabilities. Psychonomic Monograph Supplements, 1972, 4, Whole No. 65.

Pike, A. R. Response latency models for signal detection. Psychological Review, 1973, 80, 53-68.

Serular, R. W., \& Abrams, M. Visual sameness: A choice time analysis of pattern recognition processes. Journal of Experimental Psychology, 1968, 77, 232-238.

ShePaRd, R. N. Attention and the metric structure of the stimulus space. Journal of Mathematical Psychology, 1964, 1, 54-87.

Shepard, R. N., \& Cermak, G. W. Perceptual-cognitive explorations of a toroidal set of free-form stimuli. Cognitive Psychology, 1973, 4, 351-377.

Silverman, W. P., \& Goldberg, S. L. Further confirmation of same vs. different processing differences. Perception \& Psychophysics, 1975, 17, 189-193.

TAYLOR, D. A. Effect of identity in the multiletter matching task. Journal of Experimental Psychology: Human Perception and Performance, 1976, 2, 417-428.

(Manuscript received January 25, 1982; revision accepted for publication April 22, 1982.) 